\title{
CORRECTION TO A PAPER BY A. G. PAKES CHRISTIAN BERG
}

(Received 25 March 2002; revised 8 January 2003)

Communicated by V. T. Stefanov

\begin{abstract}
Starting from a probability $\sigma$ on the half-line with moments of any order A. G. Pakes has defined probabilities $\sigma_{r}$ by length biasing of order $r$ and $g_{r}$ by the stationary-excess operation of order $r$, $r=1,2, \ldots$. Examples are given to show that $\sigma$ can be determined in the Stieltjes sense while $\sigma_{1}$ and $g_{1}$ are indeterminate in the Stieltjes sense. This shows that a statement in a recent paper by Pakes does not hold.
\end{abstract}

2000 Mathematics subject classification: primary 44A60; secondary 60E05.

Keywords and phrases: Indeterminate moment problems, length biasing, stationary excess-operation.

\section{Introduction}

In a recent paper [11] Pakes is considering the criteria of Carleman and Krein together with some converse results. We shall use the notation of [11]. For a measure $\sigma$ on the half-line $\mathbb{R}_{+}$with moments of any order and distribution function $F$, Pakes introduces the measure $\sigma_{r}$ with distribution function $F_{r}$ given by

$$
F_{r}(x)=\mu_{r}^{-1} \int_{0}^{x} v^{r} d F(v),
$$

where $\left\{\mu_{n}\right\}$ is the moment sequence of $F$. The moment sequence of $F_{r}$ is $\mu_{n}(r)=$ $\mu_{r+n} / \mu_{r}$. The construction is called length biasing of order $r$, and $r$ can be any non-negative integer.

In [11, page 92] Pakes remarks: 'Obviously $\left\{\mu_{n}\right\}$ is S-determining if and only if $\left\{\mu_{n}(r)\right\}$ is.'

This is not true. While it is clear indeed that S-indeterminacy of $\sigma$ implies Sindeterminacy of $\sigma_{r}$, the converse is false.

(C) 2004 Australian Mathematical Society $1446-7887 / 04 \$ A 2.00+0.00$ 
In fact, in our paper with Thill [7] we completely characterized the probabilities $\sigma$ on the half-line which are S-determinate but for which $\sigma_{1}$ is not S-determinate. This characterization was the starting point for the solution of the Challifour problem solved in [7].

This lead us in [7] to introduce an index of determinacy:

For a measure $\sigma$ on the half-line with moments of any order and which is Sdeterminate $(\operatorname{det}(\mathrm{S})$ in short) the index (of determinacy) of $\sigma$ is

$$
\operatorname{ind}(\sigma)=\sup \left\{r \in \mathbb{N}_{0} \mid \sigma_{r} \text { is } \operatorname{det}(S)\right\} .
$$

Theorems 5.5 and 5.6 of [7] contain a complete characterization of the measures with ind $(\sigma)=k$. In a continuation [6] we considered the relation between the index and the denseness of the polynomials in $L^{2}$-spaces.

In later papers with Duran $[4,5]$ we extended this to the Hamburger case, that is, for measures on the real line with moments of any order. For a survey of these results see [2]. It should be added that the remark of Pakes is true if $\sigma$ is a non-discrete measure, because such a measure is either S-indeterminate or S-determinate with ind $(\sigma)=\infty$. Our observation has also the consequence that $\sigma$ can be S-determinate although the stationary-excess operation of order 1 defined in [11] leads to an S-indeterminate probability density

$$
g_{1}(x)=\bar{F}(x) / \mu_{1}, \quad \bar{F}(x)=1-F(x) .
$$

In particular, the first part of Theorem 5 in [11] is not true:

THEOREM 1.1. There exists $S$-determinate measures $\sigma$ for which

$$
\int_{x^{\prime}}^{\infty} x^{-3 / 2}(-\log \bar{F}(x)) d x<\infty, \quad x^{\prime}>0,
$$

and the density $g_{1}(x)$ is $S$-indeterminate.

We shall explain why the result fails and also give a concrete counterexample in the next section.

\section{Counterexamples}

For the general theory of the moment problem see [1]. Let us first recall that if $\sigma$ is S-indeterminate, there are infinitely many solutions to the corresponding Stieltjes moment problem. Among those are the $\mathrm{N}$ (evanlinna)-extremal solutions $\nu_{t}$ supported by $[0, \infty[$. Here the parameter $t$ can be any real number in a well-defined interval 
$[\alpha, 0]$ where $\alpha<0$, see [8, page 179] for details. The particular value $t=0$ gives a measure of the form

$$
\nu_{0}=\beta_{0} \varepsilon_{0}+\sum_{n=1}^{\infty} \beta_{n} \varepsilon_{x_{n}},
$$

where the masses $\beta_{n}>0$ sum to 1 and $0<x_{1}<x_{2}<\cdots$ tend to infinity. If the mass at zero is removed from $\nu_{0}$, and we rescale to a probability $\sigma$, that is,

$$
\sigma=\left(\nu_{0}-\beta_{0} \varepsilon_{0}\right) /\left(1-\beta_{0}\right),
$$

then $\sigma$ is S-determinate and determinate even for the corresponding Hamburger moment problem. For different proofs of this see [1, page 115] and [3]. Let as before $\left\{\mu_{n}\right\}$ be the moment sequence of $\sigma$.

The probability measure of length biasing of order 1

$$
\sigma_{1}=\frac{t}{\mu_{1}} d \sigma(t)
$$

is indet( $\mathrm{S})$ because $\sigma_{1}$ is proportional to $t d \nu_{0}(t)$, which is clearly indet( $\left.\mathrm{S}\right)$ because $\nu_{0}$ is so.

Let $F$ be the distribution function of $\sigma$ and define $\bar{F}(x)=1-F(x), g_{1}(x)=$ $\bar{F}(x) / \mu_{1}$.

Then $g_{1}$ is a probability density with moments of any order and moment sequence

$$
\bar{\mu}_{n}(1)=\frac{1}{1+n} \frac{\mu_{n+1}}{\mu_{1}} \text {. }
$$

We claim that $g_{1}$ is indet(S), because it is the product of the $S$-indeterminate sequence $\mu_{n+1} / \mu_{1}$ with the moment sequence of Lebesgue measure on $[0,1]$, see Lemma 2.1 below.

As a preparation for Lemma 2.1 we shall recall the Mellin transformation.

The (open) positive half-line is a locally compact abelian group under multiplication, and the Mellin transformation is the Fourier transformation in the sense of harmonic analysis on such groups.

The corresponding convolution of measures is denoted $\diamond$, so $\tau \diamond \chi$ is the image measure under $(x, y) \mapsto x y$ of the product measure $\tau \otimes \chi$. The Mellin transformation $\mathscr{M}$ is defined for finite (complex) measures by

$$
\mathscr{M}(\tau)(x)=\int_{0}^{\infty} t^{i x} d \tau(t), \quad x \in \mathbb{R} .
$$

The Mellin transform of the convolution product is the ordinary product of the Mellin transforms. Furthermore, for the $n$ 'th moments we have $\mu_{n}(\tau \diamond \chi)=$ $\mu_{n}(\tau) \mu_{n}(\chi)$. 
The Mellin transform of the Lebesgue measure $m$ on the unit interval $[0,1]$ is

$$
\mathscr{M}(m)(x)=\frac{1}{1+i x},
$$

hence non-vanishing. The Mellin transformation is one-to-one which implies the first statement of Lemma 2.1 .

LEMMA 2.1. The mapping $\tau \mapsto \tau \diamond m$ is one-to-one. If $\tau$ is indet(S), then so is $\tau \diamond m$.

PROOF. The second statement follows from the first, because if $\tau$ and $\chi$ are different positive measures with the same moments, then $\tau \diamond m$ and $\chi \diamond m$ are different, and they also have identical moments.

REMARK 2.2. There exists a measure $\tau$ which is $\operatorname{det}(\mathrm{S})$ and yet $\tau \diamond m$ is indet(S).

The measure $\nu_{0}$ from (2) can be written $\nu_{0}=\beta_{0} \varepsilon_{0}+\rho$ and $\nu_{0} \diamond m=\beta_{0} \varepsilon_{0}+\rho \diamond m$ is indet(S) by Lemma 2.1. Since $\rho \diamond m$ is absolutely continuous we can conclude that $\rho \diamond m$ is indeterminate. In fact, if $\rho \diamond m$ was determinate, then the polynomials are dense in $L^{2}(\rho \diamond m)$ and hence in $L^{2}\left(\nu_{0} \diamond m\right)$ by [3, Lemma 2]. Therefore the indeterminate measure $\nu_{0} \diamond m$ is $\mathrm{N}$-extremal, but this contradicts the fact that it is non-discrete.

The probability $\tau=\rho /\left(1-\beta_{0}\right)(=\sigma$ from (3)) satisfies the claim of the remark.

The author does not know if the phenomenon of Remark 2.2 can hold if $\tau$ is non-discrete or absolutely continuous.

REMARK 2.3. The Krein condition (1) cannot distinguish between the measures $\nu_{0}$ and $\sigma$ given by (2) and (3).

If we let $F$ and $G$ denote the corresponding distribution functions, condition (1) for $\bar{F}$ takes the form

$$
2 \sum_{n=N}^{\infty}-\log \left(1-\beta_{0}-\cdots-\beta_{n}\right)\left(\frac{1}{\sqrt{x_{n}}}-\frac{1}{\sqrt{x_{n+1}}}\right)<\infty,
$$

while for $\bar{G}$ it has the form

$$
2 \sum_{n=N}^{\infty}-\log \left(\frac{1-\beta_{0}-\cdots-\beta_{n}}{1-\beta_{0}}\right)\left(\frac{1}{\sqrt{x_{n}}}-\frac{1}{\sqrt{x_{n+1}}}\right)<\infty .
$$

Since

$$
\sum_{n=N}^{\infty}\left(\frac{1}{\sqrt{x_{n}}}-\frac{1}{\sqrt{x_{n+1}}}\right)=\frac{1}{\sqrt{x_{N}}}
$$


the two series in (4), (5) converge simultaneously, and we know that $\nu_{0}$ is indet(S), but $\sigma$ is $\operatorname{det}(\mathrm{S})$.

We shall now give a concrete example of a probability of the form (2), which leads to a probability $\sigma$ which is $\operatorname{det}(S)$ and for which the Krein condition (1) nevertheless holds by direct verification. This gives a concrete example showing that the first part of Theorem 5 in [11] is not correct.

The example comes from a birth and death process with quartic rates studied by Berg and Valent, see $[9,8]$.

A birth and death process is defined by the sequences $\left(\lambda_{n}\right)_{n \geq 0}$ of birth rates and $\left(\mu_{n}\right)_{n \geq 0}$ of death rates, restricted by $\lambda_{n}>0, \mu_{n+1}>0$ for $n \geq 0$ and $\mu_{0} \geq 0$, see for example [10].

In order to solve the so-called Kolmogorov equation, one studies the polynomials $F_{n}(x)$ defined by the recurrence

$$
\left(\lambda_{n}+\mu_{n}-x\right) F_{n}(x)=\mu_{n+1} F_{n+1}(x)+\lambda_{n-1} F_{n-1}(x), \quad n \geq 0
$$

with the initial conditions $F_{-1}(x)=0, F_{0}(x)=1$.

Defining

$$
\pi_{0}=1, \quad \pi_{n}=\frac{\lambda_{0} \cdots \lambda_{n-1}}{\mu_{1} \cdots \mu_{n}}, \quad n \geq 1
$$

and

$$
a_{n}=\lambda_{n}+\mu_{n}, \quad b_{n}=\sqrt{\lambda_{n} \mu_{n+1}}, \quad n \geq 0,
$$

it is well known that the polynomials

$$
P_{n}(x)=(-1)^{n} \frac{1}{\sqrt{\pi_{n}}} F_{n}(x)
$$

satisfy the three term recurrence relation

$$
x P_{n}(x)=b_{n} P_{n+1}(x)+a_{n} P_{n}(x)+b_{n-1} P_{n-1}(x), \quad n \geq 1
$$

together with the initial conditions $P_{0}(x)=1, P_{1}(x)=\left(x-a_{0}\right) / b_{0}$.

By Favard's Theorem the polynomials $\left\{P_{n}\right\}$ form an orthonormal system with respect to some probability measure on the half-line and the corresponding moment sequence is a Stieltjes moment sequence.

We shall consider the following quartic rates

$$
\lambda_{n}=(4 n+1)(4 n+2)^{2}(4 n+3), \quad \mu_{n}=(4 n-1)(4 n)^{2}(4 n+1), \quad n \geq 0
$$

initially considered in $[14,12,13]$. Note that $\mu_{0}=0$ and

$$
\pi_{n}=\frac{1}{4 n+1}\left(\frac{(1 / 2)_{n}}{n !}\right)^{2} \sim \frac{1}{4 \pi} \frac{1}{n^{2}}, \quad \lambda_{n-1} \pi_{n-1}=\mu_{n} \pi_{n} \sim \frac{64}{\pi} n^{2},
$$


and it follows from known criteria that the corresponding moment problem is indet( $\mathrm{S})$, see for example [9].

The $\mathrm{N}$-extremal measure $\nu_{0}$ is given by

$$
\nu_{0}=\frac{\pi}{K_{0}^{2}} \varepsilon_{x_{0}}+\frac{4 \pi}{K_{0}^{2}} \sum_{n=1}^{\infty} \frac{2 n \pi}{\sinh (2 n \pi)} \varepsilon_{x_{n}}, \quad x_{n}=\left(\frac{2 n \pi}{K_{0}}\right)^{4},
$$

and the constant $K_{0}$ is given by en elliptic integral, see [9].

From the general theory mentioned above

$$
\sigma=c \sum_{n=1}^{\infty} \frac{2 n \pi}{\sinh (2 n \pi)} \varepsilon_{x_{n}}
$$

is determinate. The normalization constant $c$ (expressible by $K_{0}$ ) is chosen so that $\sigma$ is a probability. The function $\bar{F}$ is piecewise constant and to establish (1), we have to prove that

$$
\sum_{n=1}^{\infty}-\log \left(y_{n}\right)\left(\frac{1}{\sqrt{x_{n}}}-\frac{1}{\sqrt{x_{n+1}}}\right)<\infty,
$$

where $x_{n}$ is as above and

$$
y_{n}=c \sum_{k=n+1}^{\infty} \frac{2 k \pi}{\sinh (2 k \pi)} \text {. }
$$

Using

$$
y_{n} \geq c \int_{n+1}^{\infty} \frac{2 x \pi}{\sinh (2 x \pi)} d x \geq 4 \pi c \int_{n+1}^{\infty} x e^{-2 \pi x} d x \geq 2 c(n+1) e^{-2 \pi(n+1)},
$$

we see that (6) holds.

\section{References}

[1] N. I. Akhiezer, The classical moment problem and some related questions in analysis (Oliver and Boyd, Edinburgh, 1965).

[2] C. Berg, 'Recent results about moment problems', in: Probability Measures on Groups and Related Structures XI, Proceedings Oberwolfach 1994 (ed. H. Heyer) (World Scientific, Singapore, 1995).

[3] C. Berg and J. P. R. Christensen, 'Density questions in the classical theory of moments', Ann. Inst. Fourier 31 (1981), 99-114.

[4] C. Berg and A. J. Duran, 'The index of determinacy for measures and the $l^{2}$-norm of orthonormal polynomials', Trans. Amer. Math. Soc. 347 (1995), 2795-2811.

[5] — ' 'When does a discrete differential perturbation of a sequence of orthonormal polynomials belong to $\ell^{2}$ ?', J. Funct. Anal. 136 (1996), 127-153. 
[6] C. Berg and M. Thill, 'A density index for the Stieltjes moment problem', in: Orthogonal polynomials and their applications (eds. C. Brezinski, L. Gori and A. Ronveaux), IMACS Ann. Comput. Appl. Math. 9 (Baltzer, Basel, 1991) pp. 185-188.

[7] —. 'Rotation invariant moment problems', Acta Math. 167 (1991), 207-227.

[8] C. Berg and G. Valent, 'The Nevanlinna parametrization for some indeterminate Stieltjes moment problems associated with birth and death processes', Methods and Applications of Analysis 1 (1994), 169-209.

[9] _- 'Nevanlinna extremal measures for some orthogonal polynomials related to birth and death processes', J. Comp. Appl. Math. 57 (1995), 29-43.

[10] M. E. H. Ismail, J. Letessier, D. Masson and G. Valent, 'Birth and death processes and orthogonal polynomials', in: Orthogonal polynomials: theory and practice, NATO ASI series C 294 (Kluwer Academic Publishers, The Netherlands, 1990) pp. 229-255.

[11] A. G. Pakes, 'Remarks on the converse Carleman and Krein criteria for the classical moment problem', J. Austral. Math. Soc. 71 (2001), 81-104.

[12] G. Valent, 'Orthogonal polynomials for a quartic birth and death process', in: Proceedings of the Granada conference 1991, J. Comput. Appl. Math. 49 (North Holland, Amsterdam, 1993) pp. 281-288.

[13] — 'Asymptotic analysis of some associated orthogonal polynomials connected with elliptic functions', SIAM J. Math. Anal. 25 (1994), 749-775.

[14] —, 'Exact solutions of some quadratic and quartic birth and death processes and related orthogonal polynomials', J. Comput. Appl. Math. 67 (1996), 103-127.

\section{Universitetsparken 5}

DK-2100 København $\emptyset$

Denmark

e-mail: berg@math.ku.dk 
